PUPT-2107

hep-th/0401041

\title{
Integrable Open Spin Chains in Defect Conformal Field Theory
}

\author{
Oliver DeWolfe ${ }^{a}$ and Nelia Mann ${ }^{b}$ \\ ${ }^{a}$ Department of Physics, Princeton University, \\ Princeton, NJ 08544 \\ odewolfe@princeton.edu \\ ${ }^{b}$ Department of Physics, University of California, \\ Santa Barbara, CA 93106 \\ nelia@physics.ucsb.edu
}

\begin{abstract}
We demonstrate that the one-loop dilatation generator for the scalar sector of a certain perturbation of $\mathcal{N}=4$ Super Yang-Mills with fundamentals is the Hamiltonian of an integrable spin chain with open boundary conditions. The theory is a supersymmetric defect conformal field theory (dCFT) with the fundamentals in hypermultiplets confined to a codimension one defect. We obtain a $\mathcal{K}$-matrix satisfying a suitably generalized form of the boundary Yang-Baxter equation, study the Bethe ansatz equations, and demonstrate how Dirichlet and Neumann boundary conditions arise in field theory, and match to existing results in the plane wave limit.
\end{abstract}




\section{Introduction}

$\mathcal{N}=4$ Super Yang-Mills theory in four dimensions has a number of fascinating properties. It is exactly superconformal. In the case of an $S U(N)$ gauge group (to which we restrict ourselves here) it is believed to be precisely dual to Type IIB string theory in an $A d S_{5} \times S^{5}$ background with $N$ units of five-form flux [1], 2], 3], 4]. This duality becomes more tractable to study in the limit of excitations with large $R$-charge, where the gravity dual geometry reduces to a maximally supersymmetric plane wave, as investigated by Berenstein, Maldacena, and Nastase [5]. In this regime it is particularly easy to see how a closed string arises as a collection of "bits," identified in the dual as fields in a gauge invariant single-trace operator.

More recently it has emerged that an integrable structure is present on both sides of this duality. The planar one-loop matrix of anomalous dimensions for gauge invariant local operators has been shown to be the Hamiltonian of an integrable spin chain; the case of scalar operators was demonstrated by Minahan and Zarembo [6], and their arguments were generalized to all gauge invariant local operators by Beisert and Staudacher [7]. Furthermore, on the string theory side the worldsheet Green-Schwarz theory was shown by Bena, Polchinski, and Roiban [8] to possess an infinite number of nonlocal conserved quantities. The two manifestations of integrability were related by Dolan, Nappi, and Witten [9]; more recent work on integrability and $A d S / \mathrm{CFT}$ includes [10]-28].

One natural question to ask is whether the integrability of $\mathcal{N}=4 \mathrm{SYM}$ is present in other field theories. Systems closely related to $\mathcal{N}=4$ are the most likely to share in this special property; for other results on this question see [29], 30]. A particularly interesting feature to introduce is fundamental matter, associated with open strings in the gravity dual.

One model with these properties is the defect conformal field theory (dCFT) formulated in [31]; for more work on defect field theories see 32- 47]. This system couples the fourdimensional fields and dynamics of $\mathcal{N}=4 \mathrm{SYM}$ to $M$ new three-dimensional hypermultiplets localized on a flat codimension one hypersurface, or "defect." These hypermultiplets transform in the fundamental representation of the three-dimensional part of the $\mathcal{N}=4$ gauge field, and break half the supersymmetry. There is only one free parameter, the bulk gauge coupling, and interestingly, the theory is exactly superconformal [31], [32]. For $M \ll N$, the gravity dual is IIB string theory on $A d S_{5} \times S^{5}$, with the new ingredient of $M$ D5-branes wrapped on $A d S_{4} \times S^{2}$ [48, [4]. ${ }^{1}$ Open string modes on the D5-brane are dual to gaugeinvariant operators localized on the defect, which have the form of a number of bulk adjoint fields between a pair of fundamental defect fields; the defect fields thus play the role of the ends of the open string.

In this paper, we consider the question of whether this dCFT is integrable, as $\mathcal{N}=4 \mathrm{SYM}$

\footnotetext{
${ }^{1}$ For $M \sim N$ the backreaction of the D5-branes must be taken into account, and the supergravity solution is unknown; but see [50], [51].
} 
is. We study the sector of gauge-invariant, local defect operators composed of Lorentz singlet fields, generalizing the approach of [6]. After calculating the one-loop anomalous dimension matrix in the planar limit for this class of operators, we search for an integrable system with this operator as the Hamiltonian. This comes down to finding integrable boundary conditions for the $S O(6)$ spin chain of [6], which can be codified in a matrix $\mathcal{K}$ that solves the boundary Yang-Baxter equation, suitably generalized to incorporate boundary flavor degrees of freedom. ${ }^{2}$ We find such a $\mathcal{K}$-matrix and demonstrate that the associated Hamiltonian indeed matches the one-loop dilatation generator of the dCFT, demonstrating integrability for our sector of operators. We conjecture that this integrability persists to the complete set of one-loop gauge invariant operators.

Having obtained the integrable structure, one may use the Bethe ansatz to diagonalize the anomalous dimension matrix. Studying single excitations on the open chain and their reflections off the boundary, we demonstrate that they obey either Dirichlet or Neumann boundary conditions, matching the possible excitations of the dual open string. Furthermore, the periodicities and phase shifts conspire so that the the propagation of left- and rightmovers on the chain is equivalent to that of only left-movers on an effective periodic closed chain of twice the length - the familiar so-called doubling trick of open string theory. The integrability of the system thus provides a window through which we may see more directly how open strings are realized within the dual dCFT. We also compare our results to the results of Lee and Park in the plane-wave limit [33], and find agreement.

In section 2 we review the defect conformal field theory and calculate the planar one-loop matrix of anomalous dimensions. We solve the boundary Yang-Baxter equation to identify the integrable structure in section 3, and formulate the Bethe ansatz and consider single excitations in section 4. In section 5 we conclude. Field theory conventions are listed in an appendix.

Note added. As this paper was being finalized there appeared work by Chen, Wang, and $\mathrm{Wu}$ [54] that obtains apparently similar results for a different deformation of $\mathcal{N}=4 \mathrm{SYM}$ with fundamentals. Interesting earlier work on open spin chains in gauge theories with matter appears in [55]-60].

\section{Field Theory Computations}

\subsection{Review of Defect Conformal Field Theory}

The defect theory we study [31] is a descendant of $\mathcal{N}=4$ Super-Yang-Mills theory with $S U(N)$ gauge group. The $\mathcal{N}=4$ theory contains a gauge field $A_{\mu}$, and adjoint Majorana spinors $\lambda^{\alpha}$ and real scalars $X^{i}$ in the 4 and 6 respectively of the $S O(6) \mathrm{R}$-symmetry. The

\footnotetext{
${ }^{2}$ For work on classification of integrable boundary conditions without this generalization, see [52], [53].
} 
dCFT introduces $M$ additional 3D hypermultiplets propagating on the hypersurface $x_{3}=0$. The new fields break the total symmetry group from $P S U(2,2 \mid 4)$ down to $O S p(4 \mid 4)$, where the $4 \mathrm{D}$ conformal group $S O(4,2)$ is reduced to the $3 \mathrm{D}$ conformal group $S O(3,2)$, the Rsymmetry is broken $S O(6)_{R} \rightarrow S O(3)_{H} \times S O(3)_{V}$, and the supersymmetry is cut in half. The defect fields are $M$ complex scalars $q^{m}$ transforming in the $(\mathbf{2}, \mathbf{1})$ of $S O(3)_{H} \times S O(3)_{V}$ and $M$ complex 2-component fermions $\Psi^{a}$ transforming in the $(\mathbf{1}, \mathbf{2})$. Conventions and the action for $\mathcal{N}=4 \mathrm{SYM}$ can be found in the appendix.

The bulk fields are arranged with respect to the preserved $3 \mathrm{D}$ symmetry group into a vector multiplet $\left\{A_{k}, P_{+} \lambda^{\alpha}, X_{V}^{A}, D_{3} X_{H}^{I}\right\}$ and a hypermultiplet $\left\{A_{3}, P_{-} \lambda^{\alpha}, X_{H}^{I}, D_{3} X_{V}^{A}\right\}$, where $k=0,1,2, A=1,2,3$, and $I=4,5,6 . P_{+} \lambda$ and $P_{-} \lambda$ with $P_{ \pm} \equiv\left(1 \pm \gamma^{5} \gamma^{3}\right) / 2$ indicate the splitting of a 4D Majorana spinor into two 3D Majorana spinors. The $X_{H}$ and $X_{V}$ scalars transform as $(\mathbf{3}, \mathbf{1})$ and $(\mathbf{1}, \mathbf{3})$ of $S O(3)_{H} \times S O(3)_{V}$, while the $\lambda^{\alpha}$ are arranged into a $(\mathbf{2}, \mathbf{2})$ field $\lambda_{a m}$.

The defect fields couple directly only to the bulk vector multiplet. The total action for the theory is that of $\mathcal{N}=4 \mathrm{SYM}$ together with

$$
\begin{aligned}
S_{3}= & S_{k i n}+S_{y u k}+S_{p o t} \\
S_{k i n}= & \frac{1}{g^{2}} \int d^{3} x\left(\left(D^{k} q^{m}\right)^{\dagger} D_{k} q^{m}-i \bar{\Psi}^{a} \rho^{k} D_{k} \Psi^{a}\right) \\
S_{y u k}= & \frac{1}{g^{2}} \int d^{3} x\left(i \bar{\Psi}^{a} P_{+} \lambda_{a m} q^{m}-i \bar{q}^{m} \bar{\lambda}_{m a} P_{+} \Psi^{a}+\bar{\Psi}^{a} \sigma_{a b}^{A} X_{V}^{A} \Psi^{a}\right), \\
S_{p o t}= & \frac{1}{g^{2}} \int d^{3} x\left(\bar{q}^{m} X_{V}^{A} X_{V}^{A} q^{m}+i \epsilon_{I J K} \bar{q}^{m} \sigma_{m n}^{I} X_{H}^{J} X_{H}^{K} q^{n}\right) \\
& +\frac{1}{g^{2}} \int d^{3} x\left(\bar{q}^{m} \sigma_{m n}^{I}\left(D_{3} X_{H}^{I}\right) q^{n}+\frac{1}{4} \delta(0) \operatorname{Tr}\left(\bar{q}^{m} \sigma_{m n}^{I} q^{n}\right)^{2}\right),
\end{aligned}
$$

with $D_{k} q=\partial_{k} q-i A_{k} q$ and similarly for $\Psi$. The total theory has only one coupling $g$, which is exactly marginal [32. We have written (11) - (41) for $M=1$, which is sufficient for our purposes; for $M>1$, dual to multiple D5-branes, flavor indices appear on $q$ and $\Psi$ in an obvious way.

\subsection{Anomalous Dimensions for Scalar Operators}

We are interested in local, gauge-invariant defect operators constructed out of Lorentz scalar fields that are "single-trace" in the sense that one line of color runs through the entire operator. These have the form

$$
\mathcal{O}=\psi_{m, j_{1}, \ldots j_{L}, n} \bar{q}_{m} X^{j_{1}} \ldots X^{j_{L}} q^{n}
$$

with the obvious sum over color indices implied. We can think of each operator as an open spin chain consisting of vector spaces $W_{\bar{\alpha}} \times V_{1} \times \ldots \times V_{L} \times W_{\beta}$, with $V_{i}$ in the $\mathbf{6}$ of $S O(6)$, $\beta$ in the $\mathbf{2}$ of $S O(3)_{H}$, and $\bar{\alpha}$ in the $\overline{\mathbf{2}}{ }^{3}$

\footnotetext{
${ }^{3}$ The $\overline{\mathbf{2}}$ is equivalent to the $\mathbf{2}$, but we write it this way to avoid worrying about epsilon symbols.
} 
We are interested in the one-loop planar contributions to the matrix of anomalous dimensions for these operators. We calculate this by evaluating the correlation function

$$
\Gamma \equiv\left\langle\bar{q}_{n^{\prime}}\left(z_{\beta}\right) X^{i_{L}}\left(z_{L}\right) \ldots X^{i_{1}}\left(z_{1}\right) q^{m^{\prime}}\left(z_{\alpha}\right) \mathcal{O}(0)\right\rangle
$$

Only nearest neighbor fields along the chain will interact to this order, reducing the contributions to effective correlators of two fields with a two-site piece of the operator $\mathcal{O}$.

First consider the interaction between two bulk scalars $X^{i_{a}}$ and $X^{i_{b}}$. Interactions that exist purely in the $\mathcal{N}=4$ theory were calculated for the case of the closed spin chain [6] (see also [5], 61, 62, 63]) and include the nontrivial interactions of gauge boson exchange and the 4-point $X$ interaction, as well as contributions of the one-loop wavefunction renormalization of each $X$ field. In principle, additional interactions involving the defect fields can contribute as well. However, one can quickly convince oneself by looking at the action (11) that there are no nontrivial defect interactions between $4 X$ 's at lowest order. Furthermore, although diagrams with defect fields contributing to $\langle X X\rangle$ do exist, they are forbidden on symmetry grounds from leading to a divergent piece in the $X$ self-energy [31.

Consequently, the contribution of two $X$ fields to the matrix of anomalous dimension is unchanged from the pure $\mathcal{N}=4$ case. As a result, all the interactions in $\Gamma$ not involving a defect field match the contributions in the case of the closed chain. (This also implies that the anomalous dimension matrix for closed chains of $X$ 's in the defect theory takes exactly

the same form as in $\mathcal{N}=4 \mathrm{SYM}$.) The defect interactions are localized to the ends of the chain and will have the interpretation of integrable boundary conditions added to an already integrable system.

\subsection{Differential Regularization and Bulk Interactions}

Although they are well known [6], we include the calculation of the $X X$ interactions to establish our conventions. We perform our computations in position space using differential regularization 64]. The presence of the defect favors a position space regulator, and differential regularization is particularly convenient for extracting anomalous dimensions.

The essential philosophy of differential regularization is to replace amplitudes containing position-space distributions too singular to have a Fourier transform by regularized distributions differing only at the singular point; this replacement is equivalent to adding local counterterms. The regularized amplitudes have a well-defined Fourier transform but depend on an arbitrary mass scale; differentiating with respect to this scale produces the usual CallanSymanzik equations for the regulated amplitudes. As we are interested only in anomalous dimensions, we keep only logarithmic divergences, and discard quadratic divergences.

Consider first the amputated $X$ self-energy $\Gamma_{X} \equiv\left\langle X^{i}\left(z_{1}\right) X^{j}\left(z_{2}\right)\right\rangle$. There are three oneloop diagrams - fermion loop, gauge boson loop, and scalar tadpole; the last is a purely quadratic divergence and hence irrelevant for us. The fermion and gauge boson diagrams 
give

$$
\Gamma_{X}^{(1)}=g^{2} N \delta^{i j}(4-2) \partial^{\mu} \Delta_{12} \partial_{\mu} \Delta_{12}=g^{2} N \delta^{i j} \frac{8}{\left(4 \pi^{2}\right)^{2}} \frac{1}{z_{12}^{6}},
$$

where we discarded a quadratic divergence going like $\Delta_{12} \square \Delta_{12} \sim \delta\left(z_{12}\right) / z_{12}^{2}$. Regularizing according to the formulae in the Appendix, we find

$$
M \frac{\partial}{\partial M} \Gamma_{X}^{(1)}=\delta^{i j} \frac{g^{2} N}{8 \pi^{2}} \square \delta\left(z_{12}\right) .
$$

Since $\beta(g)=0$ for our dCFT, the Callan-Symanzik equation is particularly simple:

$$
\left(M \frac{\partial}{\partial M}+2 \gamma_{X}(g)\right) \Gamma_{X}=0 \text {. }
$$

Using the amputated zeroth-order result $\Gamma_{X}^{(0)}=-(1 / 2) \delta^{A B} \square \delta\left(z_{12}\right)$, we find

$$
\gamma_{X}(g)=\frac{g^{2} N}{8 \pi^{2}}
$$

To find the contribution of a pair of $X$ fields to the anomalous dimension of the defect operator, we compute the correlator of two X fields with the appropriate part of $\mathcal{O}(0)$ :

$$
\Gamma_{4} \equiv\left\langle X^{i_{1}}\left(z_{1}\right) X^{i_{2}}\left(z_{2}\right) X^{j_{1}} X^{j_{2}}(0)\right\rangle
$$

Amputating the external legs associated with $X^{i_{1}}$ and $X^{i_{2}}$, regularizing, and keeping only the divergent parts, we arrive at

$$
M \frac{\partial}{\partial M} \Gamma_{4}^{(1)}=\frac{g^{2} N^{2}}{32 \pi^{2}} \delta\left(z_{1}\right) \delta\left(z_{2}\right)\left(\left(\mathcal{T}_{A}\right)_{j_{1} j_{2}}^{i_{1} i_{2}}+\left(\mathcal{T}_{X}\right)_{j_{1} j_{2}}^{i_{1} i_{2}}+\left(\mathcal{T}_{\Sigma}\right)_{j_{1} j_{2}}^{i_{1} i_{2}}\right)
$$

where the tensor structures coming from the gauge boson exchange, 4-point $\mathrm{X}$ interaction, and self-energy between $X^{i_{1}}$ and $X^{j_{1}}$ are

$$
\left(\mathcal{T}_{A}\right)_{j_{1} j_{2}}^{i_{1} i_{2}}=\delta_{j_{1}}^{i_{1}} \delta_{j_{2}}^{i_{2}}, \quad\left(\mathcal{T}_{X}\right)_{j_{1} j_{2}}^{i_{1} i_{2}}=2 \delta_{j_{2}}^{i_{1}} \delta_{j_{1}}^{i_{2}}-\delta^{i_{1} i_{2}} \delta_{j_{1} j_{2}}-\delta_{j_{1}}^{i_{1}} \delta_{j_{2}}^{i_{2}}, \quad\left(\mathcal{T}_{\Sigma}\right)_{j_{1} j_{2}}^{i_{1} i_{2}}=-4 \delta_{j_{1}}^{i_{1}} \delta_{j_{2}}^{i_{2}}
$$

Only one of the two possible self-energies is included, so that the other may be included in the interaction of the next pair in the chain. Correspondingly, we include only one factor $\gamma_{X}$ from the Callan-Symanzik equation for the total correlator $\Gamma$ :

$$
\left(M \frac{\partial}{\partial M}+\gamma_{X}+\gamma_{\mathcal{O}}\right) \Gamma_{4}=0
$$

Using this equation, the zero-loop expression $\Gamma_{4}^{(0)}=N \delta_{j_{1}}^{i_{1}} \delta_{j_{2}}^{i_{2}} \delta\left(z_{1}\right) \delta\left(z_{2}\right)$, and the result (10) for $\gamma_{X}$, we obtain

$$
\left(\gamma_{\mathcal{O}}\right)_{j_{1} j_{2}}^{i_{1} i_{2}}=\frac{g^{2} N}{16 \pi^{2}}\left\{2 \delta_{j_{1}}^{i_{1}} \delta_{j_{2}}^{i_{2}}-2 \delta_{j_{2}}^{i_{1}} \delta_{j_{1}}^{i_{2}}+\delta^{i_{1} i_{2}} \delta_{j_{1} j_{2}}\right\}
$$

Summed over all nearest neighbor pairs, (15) forms the complete one-loop planar dilatation operator for a closed chain [6]. 


\subsection{Defect Interactions}

We turn now to the defect interactions. One necessary ingredient is the $q$ self-energy. Defining $\Gamma_{q} \equiv\left\langle q^{m}\left(y_{1}\right) \bar{q}_{n}\left(y_{2}\right)\right\rangle$, we find 4 diagrams at one-loop order: a $\Psi / \lambda$ fermion loop, a $\partial_{3} X_{H}$ loop, a gauge boson loop, and a $q$ tadpole; the last diagram does not contribute to the logarithmic divergence. The contributions to the amputated self-energy are

$$
\begin{aligned}
\Gamma_{q, \Psi / \lambda}^{(1)} & =2 g^{2} N \delta_{n}^{m} \operatorname{Tr}\left(S_{12} \hat{s}_{12}\right), \\
\Gamma_{q, \partial_{3} X_{H}}^{(1)} & =-\left.\frac{3 g^{2} N}{2} \delta_{n}^{m} \mathcal{D}_{12}\left(\partial_{x}^{2} \Delta_{12}\right)\right|_{x=0}, \\
\Gamma_{q, A_{\mu}}^{(1)} & =-g^{2} N \delta_{n}^{m}\left(2 \partial^{k} \mathcal{D}_{12} \partial_{k} \Delta_{12}+2 \Delta_{12} \nabla^{2} \mathcal{D}_{12}+\frac{1}{2} \mathcal{D}_{12} \nabla^{2} \Delta_{12}\right),
\end{aligned}
$$

leading to the total logarithmic divergence:

$$
\Gamma_{q}^{(1)}=\frac{g^{2} N}{(4 \pi)\left(4 \pi^{2}\right)} \delta_{n}^{m}(8+3-5) \frac{1}{\left|y_{12}\right|^{5}} \quad \rightarrow \quad M \frac{\partial}{\partial M} \Gamma_{q}^{(1)}=\frac{g^{2} N}{4 \pi^{2}} \delta_{n}^{m} \nabla^{2} \delta\left(y_{12}\right),
$$

after regularizing according to the formulae in the appendix. The amputated zeroth-order propagator is $-\delta_{n}^{m} \nabla^{2} \delta\left(y_{12}\right)$, and hence the Callan-Symanzik equation implies

$$
\left(M \frac{\partial}{\partial M}+2 \gamma_{q}(g)\right) \Gamma_{q}=0 \quad \rightarrow \quad \gamma_{q}=\frac{g^{2} N}{8 \pi^{2}} .
$$

Next we consider the interaction at one end of the chain. We calculate

$$
\Gamma_{\partial} \equiv\left\langle X^{i}\left(z_{1}\right) q^{m^{\prime}}\left(y_{2}\right) \bar{q}_{m} X^{j}(0)\right\rangle .
$$

A universal, flavor-blind interaction is gauge boson exchange, which leads to

$$
g^{2} N^{2} \delta_{m}^{m^{\prime}} \delta^{i j}\left(\Delta_{12} \partial_{k} \Delta_{1} \partial^{k} \mathcal{D}_{2}-\frac{1}{4} \Delta_{1} \mathcal{D}_{2} \nabla^{2} \Delta_{12}+\frac{1}{2} \Delta_{1} \partial^{k} \mathcal{D}_{2} \partial_{k} \Delta_{12}-\frac{1}{2} \mathcal{D}_{2} \partial^{k} \Delta_{1} \partial_{k} \Delta_{12}\right) .
$$

If one pulls out derivatives to obtain divergence-free total derivatives plus a term with all derivatives on $\mathcal{D}_{2}$ which contains the divergence, you get

$\Gamma_{\partial, A_{\mu}}^{(1)}=$ total derivatives $+\frac{g^{2} N^{2}}{4\left(4 \pi^{2}\right)^{2}} \delta_{m}^{m^{\prime}} \delta^{i j} \delta\left(y_{2}\right) \frac{1}{z_{1}^{4}} \quad \rightarrow \quad M \frac{\partial}{\partial M} \Gamma_{\partial, A_{\mu}}^{(1)}=\frac{g^{2} N^{2}}{32 \pi^{2}} \delta_{m}^{m^{\prime}} \delta^{i j} \delta\left(z_{1}\right) \delta\left(y_{2}\right)$

There is another diagram with a scalar interaction $\bar{q} q X X$, which is different depending on whether the two $X$ scalars are $X_{V}$ or $X_{H}$. For $X^{i}=\delta_{A}^{i} X_{V}^{A}, X^{j}=\delta_{B}^{j} X_{V}^{B}$, the $q$ and $X$ scalars are charged under different $S O(3)$ s and the flavor interaction must be trivial. We find

$$
\Gamma_{\partial, X_{V}}^{(1)}=-\frac{g^{2} N^{2}}{4} \delta_{m}^{m^{\prime}} \delta^{A B} \delta\left(z_{12}\right) \Delta_{1} \mathcal{D}_{1} \quad \rightarrow \quad M \frac{\partial}{\partial M} \Gamma_{\partial, X_{V}}^{(1)}=-\frac{g^{2} N^{2}}{16 \pi^{2}} \delta_{m}^{m^{\prime}} \delta^{A B} \delta\left(z_{1}\right) \delta\left(y_{2}\right) .
$$

In the case $X^{i}=\delta_{I}^{i} X_{H}^{I}, X^{j}=\delta_{K}^{j} X_{H}^{J}$ the flavor interaction is nontrivial:

$$
\Gamma_{\partial, X_{H}}^{(1)}=-\frac{g^{2} N^{2}}{4} \delta\left(z_{12}\right)\left(i \epsilon_{I J K} \sigma_{m^{\prime} m}^{K}\right) \mathcal{D}_{2} \Delta_{2} \quad \rightarrow \quad M \frac{\partial}{\partial M} \Gamma_{\partial, X_{H}}^{(1)}=-\frac{g^{2} N^{2}}{16 \pi^{2}} \delta\left(z_{1}\right) \delta\left(y_{2}\right)\left(i \epsilon_{I J K} \sigma_{m^{\prime} m}^{K}\right) .
$$


The Callan-Symanzik equation for the complete correlator $\Gamma$ is

$$
\left(M \frac{\partial}{\partial M}+L \gamma_{X}+2 \gamma_{q}+\gamma_{\mathcal{O}}\right) \Gamma=0,
$$

where $\Gamma$ and $\gamma_{\mathcal{O}}$ are understood to be matrix-valued, while the other terms are proportional to the identity. The matrix $\gamma_{\mathcal{O}}$ can be separated into the parts acting in the bulk of the chain on two $X$ 's, and the boundary pieces. There are $L-1$ pairs of neighboring $X$ 's, each of which contributes to the matrix as (15). Notice, however, that this does not take into account one factor of $\gamma_{X}$ in (26) , as well as one contribution of the $X$ self-energy to $\Gamma$; this is because the open chain has no $X^{i_{L}} X^{i_{1}}$ interaction. Because these contributions are proportional to the identity in flavor-space, we can insert them anywhere; we find the convenient choice to be to include half the contribution of each with each boundary term.

The boundary interaction at the left end of the chain is then determined by

$$
\left(M \frac{\partial}{\partial M}+\frac{\gamma_{X}}{2}+\gamma_{q}+\gamma_{\mathcal{O}}\right) \Gamma_{q}+\frac{1}{2} M \frac{\partial}{\partial M} \Gamma_{4, \Sigma}^{(1)}=0
$$

We obtain

$$
\left(\gamma_{\mathcal{O}}\right)_{m J_{1} \ldots}^{m^{\prime} I_{1} \ldots}=\frac{g^{2} N}{16 \pi^{2}}\left(2 \delta_{m}^{m^{\prime}} \delta_{J_{1}}^{I_{1}}+2 i \epsilon_{I_{1} J_{1} K} \sigma_{m^{\prime} m}^{K}\right), \quad\left(\gamma_{\mathcal{O}}\right)_{m B_{1} \ldots}^{m^{\prime} A_{1} \ldots}=\frac{g^{2} N}{16 \pi^{2}}\left(4 \delta_{m}^{m^{\prime}} \delta_{B_{1}}^{A_{1}}\right)
$$

and analogously for the other end of the chain,

$$
\left(\gamma_{\mathcal{O}}\right)_{\ldots J_{L} n}^{\ldots I_{L} n^{\prime}}=\frac{g^{2} N}{16 \pi^{2}}\left(2 \delta_{n}^{n^{\prime}} \delta_{J_{L}}^{I_{L}}-2 i \epsilon_{I_{L} J_{L} K} \sigma_{n n^{\prime}}^{K}\right), \quad\left(\gamma_{\mathcal{O}}\right)_{\ldots B_{L} n}^{\ldots A_{L} n^{\prime}}=\frac{g^{2} N}{16 \pi^{2}}\left(4 \delta_{n}^{n^{\prime}} \delta_{B_{L}}^{A_{L}}\right)
$$

where delta-functions in the unwritten indices are understood.

In summary, we find the matrix of anomalous dimensions to be

$$
\begin{aligned}
\gamma_{\mathcal{O}} & =\frac{g^{2} N}{16 \pi^{2}}\left(\sum_{a=1}^{L-1} h_{a a+1}+\left(2 I_{\bar{\alpha} 1}+2 \bar{S}_{\bar{\alpha} 1}\right)+\left(2 I_{L \beta}+2 S_{L \beta}\right)\right), \\
h_{a a+1} & =K_{a a+1}+2 I_{a a+1}-2 P_{a a+1},
\end{aligned}
$$

where subscripts on operators indicate the vector spaces they act on, and

$$
\begin{array}{rlrl}
K_{i_{a} i_{b}}^{j_{a} j_{b}}=\delta^{j_{a} j_{b}} \delta_{i_{a} i_{b}}, & P_{i_{a} i_{b}}^{j_{a} j_{b}}=\delta_{i_{b}}^{j_{a}} \delta_{i_{a}}^{j_{b}}, & & I_{i_{a} i_{b}}^{j_{a} j_{b}}=\delta_{i_{a}}^{j_{a}} \delta_{i_{b}}^{j_{b}}, \\
S_{J n}^{I m}=-i \epsilon_{I J K} \sigma_{n m}^{K}, & S_{B n}^{A m}=\delta_{n}^{m} \delta_{B}^{A}, & S_{B n}^{I m}=S_{J n}^{A m}=0, \\
\bar{S}_{\bar{n} J}^{\bar{m} I}=i \epsilon_{I J K} \sigma_{\bar{m} \bar{n}}^{K}, & \bar{S}_{\bar{n} A}^{\bar{m} A}=\delta_{\bar{n}}^{\bar{m}} \delta_{B}^{A}, & \bar{S}_{\bar{n} B}^{\bar{m} I}=\bar{S}_{\bar{n} J}^{\bar{m} A}=0 .
\end{array}
$$

A check of our computation is that the anomalous dimensions should vanish for protected operators. In [31, the chiral primaries of the dCFT were determined to be

$$
\bar{q}^{m} \sigma_{m n}^{\left(I_{1}\right.} X_{H}^{I_{2}} X_{H}^{I_{3}} \ldots X_{H}^{\left.I_{L+1}\right)} q^{n}
$$

where parentheses denote total symmetrization and tracelessness among all the triplets of $S O(3)$. One may indeed verify that these operators are annihilated by $\gamma_{\mathcal{O}}$ as given in (30); in fact each $h_{a, a+1}$, as well as $2 I_{\bar{\alpha} 1}+2 \bar{S}_{\bar{\alpha} 1}$ and $2 I_{L \beta}+2 S_{L \beta}$, separately gives zero. 


\section{The Boundary Yang-Baxter Equation}

Having calculated the one-loop planar dilatation generator for the class of defect operators (5), we want to learn whether it is the Hamiltonian of an integrable open spin chain.

It was shown by Minahan and Zarembo in [6] that the operators composed of closed chains of $X$ fields possess this kind of integrable structure. This was done by identifying an $\mathcal{R}$-matrix, in this case the $S O(6)$-invariant

$$
\mathcal{R}_{12}(u)=\frac{1}{2}\left[u(u-2) I_{12}-(u-2) P_{12}+u K_{12}\right],
$$

where 1 and 2 label the two vector spaces acted on by the operator, and $I, P$, and $K$ are as defined in (32). These satisfy the Yang-Baxter equation

$$
\mathcal{R}_{12}(u) \mathcal{R}_{13}(u+v) \mathcal{R}_{23}(v)=\mathcal{R}_{23}(v) \mathcal{R}_{13}(u+v) \mathcal{R}_{12}(u)
$$

If we define the transfer matrix as the trace of the monodromy matrix,

$$
t(u)=\operatorname{Tr}_{a} T_{a}(u) \equiv \operatorname{Tr}_{a} \mathcal{R}_{a 1}(u) \mathcal{R}_{a 2} \cdots \mathcal{R}_{a L}(u)
$$

where $a$ is an auxiliary space and the dependence of $t(u)$ and $T_{a}(u)$ on the vector spaces $1,2, \ldots L$ comprising the chain is understood, the Yang-Baxter equation guarantees that the transfer matrices commute for arbitrary arguments:

$$
[t(u), t(v)]=0
$$

The expansion of the transfer matrix in powers of $u$ then generates an infinite number of conserved quantities, starting with the momentum and Hamiltonian. The identification of the one-loop dilatation operator for closed chains as the Hamiltonian associated with (34) demonstrated the integrability for that system.

The analogous method for introducing integrable boundary conditions was formulated in 65]; for a pedagogical summary see [66] or section 3.5 of [67. In addition to an integrable $\mathcal{R}$ -

matrix determining the dynamics for the bulk of the chain, one introduces operators $\mathcal{K}_{a}^{ \pm}(u)$ acting on either end of the spin chain. These satisfy the boundary Yang-Baxter equations, or BYBs,

$$
\begin{aligned}
\mathcal{R}_{12}(u-v) \mathcal{K}_{1}^{-}(u) \mathcal{R}_{12}(u+v) \mathcal{K}_{2}^{-}(v) & =\mathcal{K}_{2}^{-}(v) \mathcal{R}_{12}(u+v) \mathcal{K}_{1}^{-}(u) \mathcal{R}_{12}(u-v) \\
\mathcal{R}_{12}(v-u) \mathcal{K}_{1}^{+t_{1}}(u) \mathcal{R}_{12}(-u-v-2 i \gamma) \mathcal{K}_{2}^{+t_{2}}(v) & =\mathcal{K}_{2}^{+t_{2}}(v) \mathcal{R}_{12}(-u-v-2 i \gamma) \mathcal{K}_{1}^{+t_{1}}(u) \mathcal{R}_{12}(v-u)
\end{aligned}
$$

Here, $t_{i}$ refers to taking the transpose on the $i$ th vector space, and $\gamma$ is a parameter characteristic of a given $\mathcal{R}$-matrix such that

$$
\mathcal{R}_{12}^{t_{1}}(u) \mathcal{R}_{12}^{t_{1}}(-u-2 i \gamma)=\lambda(u)
$$


for some scalar function $\lambda(u)$ (for our case it is easy to see that $\gamma=2 i$ ). We can then form the open chain transfer matrices

$$
\hat{t}(u)=\operatorname{Tr}_{a} \mathcal{K}_{a}^{+}(u) T_{a}(u) \mathcal{K}_{a}^{-}(u) T_{a}^{-1}(-u) .
$$

If (35) and (38) are satisfied, these commute,

$$
[\hat{t}(u), \hat{t}(v)]=0
$$

and thus generate the family of commuting charges characteristic of an integrable system.

We must generalize this formalism slightly, from matrices $\mathcal{K}_{1}^{-}$and $\mathcal{K}_{L}^{+}$that act only on the last "ordinary" vector space in the chain, to matrices $\mathcal{K}_{\bar{\alpha} 1}^{+}$and $\mathcal{K}_{L \beta}^{-}$that also act on the degrees of freedom $W_{\bar{\alpha}}$ and $W_{\beta}$ living on the boundary. In this case the resultant $\hat{t}(u)$ acts on the vector space $W_{\bar{\alpha}} \times V_{1} \times \cdots \times V_{L} \times W_{\beta}$.

The structure of integrability is largely unaffected by this modification; the extra indices "go along for the ride" in the BYB, and (41) still holds. Given the quantum numbers of $W_{\bar{\beta}}$ and $V_{L}$, the matrix $\mathcal{K}^{-}(u)$ must take the form

$$
\begin{aligned}
\left(\mathcal{K}^{-}\right)_{\bar{n} J}^{\bar{m} I}(u) & =f(u) \delta_{\bar{n}}^{\bar{m}} \delta_{J}^{I}+g(u) \epsilon_{I J K} \sigma_{\bar{m} \bar{n}}^{K} \\
\left(\mathcal{K}^{-}\right)_{\bar{n} B}^{\bar{m} A}(u) & =h(u) \delta_{\bar{n}}^{\bar{m}} \delta_{B}^{A} \\
\left(\mathcal{K}^{-}\right)_{\bar{n} B}^{\bar{m} I}(u) & =\left(\mathcal{K}^{-}\right)_{\bar{n} J}^{\bar{m} A}(u)=0
\end{aligned}
$$

where $\bar{m}, \bar{n}$ are indices in $W_{\bar{\beta}}, I, J$ are indices in the $\mathbf{3}$ of $S O(3)_{H}$, and $A, B$ are indices in the 3 of $S O(3)_{V}$. Furthermore, if we have a $\mathcal{K}^{-}$that satisfies the first BYB equation (38), then $\left(\mathcal{K}_{1}^{+}\right)^{t_{1}}(u)=\mathcal{K}_{1}^{-}(2-u)$ satisfies the second BYB equation; however, this process will give matrices acting on a spin chain whose boundaries are both in the anti-fundamental of $S U(2)$. We can easily translate one of the boundary actions to act on the associated fundamental, and we will do so later.

Thus what we need to do is find what functions $f(u), g(u)$, and $h(u)$, if any, satisfy the first BYB. Writing out the BYB with indices, we want

$\mathcal{R}_{j_{1} j_{2}}^{i_{1} i_{2}}(u-v)\left(\mathcal{K}^{-}\right)_{\bar{n} k_{1}}^{\bar{m} j_{1}}(u) \mathcal{R}_{\ell_{1} k_{2}}^{k_{1} j_{2}}(u+v)\left(\mathcal{K}^{-}\right)_{\bar{p} \ell_{2}}^{\bar{n} k_{2}}(v)=\left(\mathcal{K}^{-}\right)_{\bar{n} j_{2}}^{\bar{m} i_{2}}(v) \mathcal{R}_{j_{1} k_{2}}^{i_{1} j_{2}}(u+v)\left(\mathcal{K}^{-}\right)_{\bar{p} k_{1}}^{\bar{n} j_{1}}(u) \mathcal{R}_{\ell_{1} \ell_{2}}^{k_{1} k_{2}}(u-v)$

There are six independent indices in this equation: $i_{1}, i_{2}, \ell_{1}, \ell_{2}$ and $\bar{m}, \bar{p}$. The first four can each take a value in either $S O(3)_{H}$ or $S O(3)_{V}$, allowing for a total of 16 equation types that need to be satisfied.

It is not hard to see that both sides will vanish whenever three of the indices $i_{1}, i_{2}, \ell_{1}, \ell_{2}$ belong to one of the $S O(3)$ 's and the fourth to the other $S O(3)$. This leaves us with 8 equations to check. Out of concern for the reader, we will give only the equations and their solution, omitting details. The case where all indices belong to the $S O(3)_{V}$ turns out to be satisfied independently of the form of the $f(u), g(u)$, and $h(u)$, as do the cases with $i_{1}, \ell_{1} \in S O(3)_{H}$ or $i_{2}, \ell_{2} \in S O(3)_{H}$. 
The same two conditions arise from either $\ell_{1}, \ell_{2} \in S O(3)_{H}$ or $i_{1}, i_{2} \in S O(3)_{H}$ :

$$
\begin{gathered}
(u+v)\left[(u-v)^{2}+2\right][h(v) f(u)-h(u) f(v)]+(u-v)\left[(u+v)^{2}+2\right][h(u) h(v)-f(u) f(v)] \\
=2(u+v+1)(u-v)(u+v-2) g(u) g(v), \\
\left(u^{2}-v^{2}\right)(u-v-2)[h(v) g(u)+h(u) g(v)]+(u+v)(u-v-2)[h(v) g(u)-h(u) g(v)] \\
-\left(u^{2}-v^{2}\right)(u+v-2)[g(u) f(v)-g(v) f(u)]-(u-v)(u+v-2)[g(u) f(v)+g(v) f(u)] \\
=-i(u+v+1)(u-v)(u+v-2) g(u) g(v)-3\left(u^{2}-v^{2}\right) g(v)(h(u)+f(u)) .
\end{gathered}
$$

The cases $i_{1}, \ell_{2}, \in S O(3)_{H}$ and $i_{2}, \ell_{1} \in S O(3)_{H}$ give two more equations:

$$
\begin{aligned}
(v-u)[f(u) f(v)-h(u) h(v)-2 g(u) g(v)] & =(v+u)[h(u) f(v)-h(v) f(u)] \\
(v-u)[f(u) g(v)+f(v) g(u)-i g(u) g(v)] & =(v+u)[h(u) g(v)-h(v) g(u)] .
\end{aligned}
$$

The case with all indices in $S O(3)_{H}$, which is the most labor-intensive, gives two final equations:

$$
\begin{array}{r}
i g(u) g(v)(u-v)[2 u+2 v-1]=2 v f(v) g(u)-2 u f(u) g(v), \\
i g(u) g(v)(u-v)(u+v-2)[2(u+v)(u-v-2)+u+v+1]= \\
2 v g(u) f(v)\left[u^{2}-v^{2}+2\right]-2 g(v) f(u)\left[u\left(u^{2}-v^{2}\right)-3\left(u^{2}-v^{2}\right)+2 u\right]- \\
3 g(v)[f(u)+h(u)]\left(u^{2}-v^{2}\right)
\end{array} .
$$

We find that

$$
f(u)=2 u^{2}-u+1, \quad g(u)=2 i u, \quad h(u)=-2 u^{2}+u+1
$$

satisfy these conditions, up to an overall function of $u$ that we fix by requiring $\mathcal{K}_{L \bar{\beta}}^{-}(0)=I_{\bar{\beta} L}$. (This overall ambiguity corresponds to reshuffling the basis of the infinite set of mutually commuting operators inside $\hat{t}(u)$.) We therefore obtain the result

$$
\begin{aligned}
\left(\mathcal{K}^{-}\right)_{\bar{n} J}^{\bar{m} I}(u) & =\left(2 u^{2}-u+1\right) \delta_{\bar{n}}^{\bar{m}} \delta_{J}^{I}+2 u i \epsilon_{I J K} \sigma_{\bar{m} \bar{n}}^{K}, \\
\left(\mathcal{K}^{-}\right)_{\bar{n} B}^{\bar{m} A}(u) & =\left(-2 u^{2}+u+1\right) \delta_{\bar{n}}^{\bar{m}} \delta_{B}^{A}, \quad\left(\mathcal{K}^{-}\right)_{\bar{n} B}^{\bar{m} I}(u)=\left(\mathcal{K}^{-}\right)_{\bar{n} J}^{\bar{m} A}(u)=0,
\end{aligned}
$$

and this implies

$$
\begin{aligned}
\left(\mathcal{K}^{+}\right)_{\bar{n} J}^{\bar{m} I}(u) & =\left(2 u^{2}-7 u+7\right) \delta_{\bar{n}}^{\bar{m}} \delta_{J}^{I}+(2 i u-4 i) \epsilon_{I J K} \sigma_{\bar{m} \bar{n}}^{K}, \\
\left(\mathcal{K}^{+}\right)_{\bar{n} B}^{\bar{m} A}(u) & =\left(-2 u^{2}+7 u-5\right) \delta_{\bar{n}}^{\bar{m}} \delta_{B}^{A}, \quad\left(\mathcal{K}^{+}\right)_{\bar{n} B}^{\bar{m} I}(u)=\left(\mathcal{K}^{+}\right)_{\bar{n} J}^{\bar{m} A}(u)=0 .
\end{aligned}
$$

Remembering that we want to deal with a spin chain having one boundary in the fundamental, we need to determine, given the above $\mathcal{K}^{-}$acting on the anti-fundamental, a $\tilde{\mathcal{K}}^{-}$that 
acts on the fundamental. For $S U(2)$, if $\psi$ is in the fundamental, then $\sigma^{2} \psi$ is in the antifundamental; therefore, we should have $\tilde{\mathcal{K}}^{-}=\sigma^{2} \mathcal{K}^{-} \sigma^{2}$. Recalling that $\sigma^{2} \sigma^{K} \sigma^{2}=-\left(\sigma^{K}\right)^{T}$, we obtain

$$
\begin{aligned}
\left(\tilde{\mathcal{K}}^{-}\right)_{n J}^{m I}(u) & =\left(2 u^{2}-u+1\right) \delta_{n}^{m} \delta_{J}^{I}-2 u i \epsilon_{I J K} \sigma_{n m}^{K} \\
\left(\tilde{\mathcal{K}}^{-}\right)_{n B}^{m A}(u) & =\left(-2 u^{2}+u+1\right) \delta_{n}^{m} \delta_{B}^{A}, \quad\left(\tilde{\mathcal{K}}^{-}\right)_{n B}^{m I}(u)=\left(\tilde{\mathcal{K}}^{-}\right)_{n J}^{m A}(u)=0 .
\end{aligned}
$$

Now, we want to form the transfer matrix

$$
\hat{t}(u)=\operatorname{Tr}_{a}\left[\mathcal{K}_{\bar{\alpha} a}^{+}(u) \mathcal{R}_{a 1}(u) \cdots \mathcal{R}_{a L}(u) \tilde{\mathcal{K}}_{a \beta}^{-}(u)\left(\mathcal{R}_{L a}(-u)\right)^{-1} \cdots\left(\mathcal{R}_{1 a}(-u)\right)^{-1}\right] .
$$

Since (as is straightforward to verify)

$$
\mathcal{R}_{b a}^{-1}(-u)=\frac{4}{\left(4-u^{2}\right)\left(1-u^{2}\right)} \mathcal{R}_{a b}(u),
$$

we find

$$
\hat{t}(u)=\operatorname{Tr}_{a}\left[\frac{4^{L}}{\left(4-u^{2}\right)^{L}\left(1-u^{2}\right)^{L}} \mathcal{K}_{\bar{\alpha} a}^{+}(u) \mathcal{R}_{a 1}(u) \cdots \mathcal{R}_{a L}(u) \tilde{\mathcal{K}}_{a \beta}^{-}(u) \mathcal{R}_{a L}(u) \cdots \mathcal{R}_{a 1}(u)\right] .
$$

We are interested in expanding $\hat{t}(u)=\sum_{n} \hat{t}_{n} u^{n}$, and identifying the Hamiltonian as $\hat{t}_{1}$. Making use of the relations

$$
\mathcal{R}_{a b}(0)=P_{a b}, \quad \frac{d}{d u} \mathcal{R}_{a b}(0)=\frac{1}{2}\left[-2 I_{a b}-P_{a b}+K_{a b}\right],
$$

and the definitions in (32), we determine that the lowest term in the expansion of $\hat{t}(u)$ is

$$
\hat{t}_{0} \equiv \hat{t}(0)=\operatorname{Tr}_{a}\left[\frac{4^{L}}{4^{L}} \mathcal{K}_{\bar{\alpha} a}^{+}(0) P_{a 1} \cdots P_{a L} I_{L \beta} P_{a L} \cdots P_{a 1}\right]=\operatorname{Tr}_{a}\left[\mathcal{K}_{\bar{\alpha} a}^{+}\right]=6 I .
$$

The next term is slightly more complicated. Breaking it into pieces, we find that

$$
\begin{array}{r}
\operatorname{Tr}_{a}\left[\partial_{u} \mathcal{K}_{\bar{\alpha} a}^{+}(0) \mathcal{R}_{a 1}(0) \cdots \mathcal{R}_{a L}(0) \tilde{\mathcal{K}}_{a \beta}^{-}(0) \mathcal{R}_{a L}(0) \cdots \mathcal{R}_{a 1}(0)\right]= \\
\operatorname{Tr}_{a}\left[\partial_{u} \mathcal{K}_{\bar{\alpha} a}^{+}(0) P_{a 1} \cdots P_{a L} I_{a \beta} P_{a L} \cdots P_{a 1}\right]=\operatorname{Tr}_{a}\left[\partial_{u} \mathcal{K}_{\bar{\alpha} a}^{+}(0)\right]=0
\end{array}
$$

and

$$
\begin{array}{r}
\operatorname{Tr}_{a}\left[\mathcal{K}_{\bar{\alpha} a}^{+}(0) \partial_{u} \mathcal{R}_{a 1}(0) \cdots \mathcal{R}_{a L}(0) \tilde{\mathcal{K}}_{a \beta}^{-}(0) \mathcal{R}_{a L}(0) \cdots \mathcal{R}_{a 1}(0)\right]+ \\
\operatorname{Tr}_{a}\left[\mathcal{K}_{\bar{\alpha} 1}^{+}(0) \mathcal{R}_{a 1}(0) \cdots \mathcal{R}_{a L}(0) \tilde{\mathcal{K}}_{a \beta}^{-}(0) \mathcal{R}_{a L}(0) \cdots \partial_{u} \mathcal{R}_{a 1}(0)\right]= \\
\frac{1}{2} \operatorname{Tr}_{a}\left[\mathcal{K}_{\bar{\alpha} 1}^{+}(0)\left(-2 I_{a 1}-P_{a 1}+K_{a 1}\right) P_{a 2} \cdots P_{a L} I_{a \beta} P_{a L} \cdots P_{a 1}\right]+ \\
\frac{1}{2} \operatorname{Tr}_{a}\left[\mathcal{K}_{\bar{\alpha} a}^{+}(0) P_{a 1} \cdots P_{a L} I_{\beta a} P_{a L} \cdots P_{a 2}\left(-2 I_{a 1}-P_{a 1}+K_{a 1}\right)\right]= \\
\operatorname{Tr}_{a}\left[\mathcal{K}_{\bar{\alpha} a}^{+}(0)\left(-2 I_{a 1}-P_{a 1}+K_{a 1}\right)\right]=12 \bar{S}_{\alpha 1}-13 I_{\alpha 1}
\end{array}
$$


The terms with the derivative acting on $\mathcal{R}_{a \ell}$ for $\ell \in 2, \ldots, L$ all behave similarly:

$$
\begin{aligned}
\operatorname{Tr}_{a}\left[\mathcal{K}_{\bar{\alpha} a}^{+}(0) \mathcal{R}_{a 1}(0) \cdots \partial_{u} \mathcal{R}_{a \ell}(0) \cdots \mathcal{R}_{a L}(0) \tilde{\mathcal{K}}_{a \beta}^{-}(0) \mathcal{R}_{a L}(0) \cdots \mathcal{R}_{a 1}(0)\right]+ \\
\operatorname{Tr}_{a}\left[\mathcal{K}_{\bar{\alpha} a}^{+}(0) \mathcal{R}_{a 1}(0) \cdots \mathcal{R}_{a L}(0) \tilde{\mathcal{K}}_{a \beta}^{-}(0) \mathcal{R}_{a L}(0) \cdots \partial_{u} \mathcal{R}_{a \ell}(0) \cdots \mathcal{R}_{a 1}(0)\right]= \\
\frac{1}{2} \operatorname{Tr}_{a}\left[\mathcal{K}_{\bar{\alpha} a}^{+}(0) P_{a 1} \cdots\left(-2 I_{a \ell}-P_{a \ell}+K_{a \ell}\right) \cdots P_{a L} I_{a \beta} P_{a L} \cdots P_{a 1}\right]+ \\
\frac{1}{2} \operatorname{Tr}_{a}\left[\mathcal{K}_{\bar{\alpha} a}^{+}(0) P_{a 1} \cdots P_{a L} I_{\beta a} P_{a L} \cdots\left(-2 I_{a \ell}-P_{a \ell}+K_{a \ell}\right) \cdots P_{a_{1}}\right]= \\
\operatorname{Tr}_{a}\left[\mathcal{K}_{\bar{\alpha} a}^{+}(0) P_{a 1} \cdots P_{a \ell-1}\left[-2 P_{a \ell}-I_{a \ell}+K_{a \ell}\right] P_{a \ell-1} \cdots P_{a 1}\right]= \\
\operatorname{Tr}_{a}\left[\mathcal{K}_{\bar{\alpha} a}^{+}(0)\right]\left(-2 P_{\ell-1 \ell}-I_{\ell-1 \ell}+K_{\ell-1 \ell}\right)=6\left(-2 P_{\ell-1 \ell}-I_{\ell-1 \ell}+K_{\ell-1 \ell}\right) .
\end{aligned}
$$

Finally, we have a term of the form

$$
\begin{aligned}
& \operatorname{Tr}_{a}\left[\mathcal{K}_{\bar{\alpha} a}^{+}(0) \mathcal{R}_{a 1}(0) \cdots \mathcal{R}_{a L}(0) \partial_{u} \tilde{\mathcal{K}}_{a \beta}^{-}(0) \mathcal{R}_{a L}(0) \cdots \mathcal{R}_{a L}(0)\right] \\
& \operatorname{Tr}_{a}\left[\mathcal{K}_{\bar{\alpha} a}^{+}(0) P_{a 1} \cdots P_{a L} \partial_{u} \tilde{\mathcal{K}}_{a \beta}^{-}(0) P_{a L} \cdots P_{a 1}\right]=\operatorname{Tr}_{a}\left[\mathcal{K}_{\bar{\alpha} a}^{+}(0) P_{a 1} \cdots \partial_{u} \tilde{\mathcal{K}}_{L \beta}^{-}(0) \cdots P_{a 1}\right]= \\
& \operatorname{Tr}_{a}\left[\mathcal{K}_{\bar{\alpha} a}^{+}(0)\right] \partial_{u} \tilde{\mathcal{K}}_{L \beta}^{-}(0)=6\left(-I_{L \beta}+2 S_{L \beta}\right) .
\end{aligned}
$$

We combine these expressions to give

$$
\hat{t}_{1}=-13 I_{\bar{\alpha} 1}+12 \bar{S}_{\bar{\alpha} 1}+\Sigma_{\ell=1}^{L-1}\left(-12 P_{\ell, \ell+1}-6 I_{\ell, \ell+1}+6 K_{\ell, \ell+1}\right)-6 I_{\beta L}+12 S_{L \beta},
$$

and adding a multiple of the identity to the result, we find

$$
\hat{t}_{1}+(25+18 L) I=6\left[\sum_{\ell=1}^{L-1} h_{\ell \ell+1}+\left(2 I_{\bar{\alpha} 1}+2 \bar{S}_{\bar{\alpha} 1}\right)+\left(2 I_{\beta L}+2 S_{L \beta}\right)\right],
$$

which is indeed proportional to the matrix of anomalous dimension $\gamma_{\mathcal{O}}$ found in (30), demonstrating that this operator is in fact a Hamiltonian for an integrable system.

\section{The Bethe Ansatz}

Having seen that the planar one-loop matrix of anomalous dimensions (30) is an integrable Hamiltonian for the spin chain, we may use the techniques of the Bethe ansatz to find the eigenvectors and eigenvalues of this matrix.

To do so one begins with a Bethe reference state, corresponding to a certain operator, and systematically creates excitations above this ground state. These excitations correspond to impurities in the chain. The "Bethe ansatz" is to assume that the eigenvectors consist of waves of impurities (or "spin waves") propagating along the chain with certain momenta $k_{i}$; integrability assures that scattering of these waves factorizes into products of two-body scattering where the magnitude of each momentum is separately conserved. 
The Bethe ansatz requires for consistency one equation for each excitation. For the closed chain, these equations are of the form

$$
e^{i k_{i} L}=\prod_{j \neq i} S_{j i}\left(k_{j}, k_{i}\right)
$$

where the $S$-matrix $S_{j i}\left(k_{j}, k_{i}\right)$ is the phase shift acquired when the $i^{\text {th }}$ excitation passes through the $j^{\text {th }}$ excitation; the equations (67) can be thought of as the requirement that the phase of an excitation as it travels all the way around the chain and comes back to itself is unity. In the special case of non-interaction, $S_{j i}=1$ and (67) reduces to the usual quantization of momentum on a circle.

In the case of systems with Lie group symmetry, extra excitations are introduced that correspond to changing the orientation in group space of the spin wave. For example, consider the general formula given in [6], eqn. (4.35):

$$
\left(\frac{u_{q, i}+i \vec{\alpha}_{q} \cdot \vec{w} / 2}{u_{q, i}-i \vec{\alpha}_{q} \cdot \vec{w} / 2}\right)^{L}=\prod_{j \neq i}^{n_{q}} \frac{u_{q, i}-u_{q, j}+i \vec{\alpha}_{q} \cdot \vec{\alpha}_{q} / 2}{u_{q, i}-u_{q, j}-i \vec{\alpha}_{q} \cdot \vec{\alpha}_{q} / 2} \prod_{q^{\prime} \neq q} \prod_{j}^{n_{q^{\prime}}} \frac{u_{q, i}-u_{q^{\prime}, j}+i \vec{\alpha}_{q} \cdot \vec{\alpha}_{q^{\prime}} / 2}{u_{q, i}-u_{q^{\prime}, j}-i \vec{\alpha}_{q} \cdot \vec{\alpha}_{q^{\prime}} / 2} .
$$

Here the $u_{q, i}$ are parameters characterizing excitations, taking the place of the $k_{i} ; i$ labels the excitation as before, while $q$ reflects the fact that the excitation can be associated to any of the simple roots $\vec{\alpha}_{q}$ of the algebra. $\vec{w}$ is the highest weight vector of the representation of the group that lives at each site. For a fundamental at each site, we will have $\vec{w}=\vec{w}^{1}$, the first fundamental weight, which has the inner product with simple roots $\vec{\alpha}_{q} \cdot \vec{w}^{1}=\delta_{q}^{1}$.

Thus for an excitation $u_{q, i}$ with $q=1$, the left hand side is

$$
\left(\frac{u_{1, i}+i / 2}{u_{1, i}-i / 2}\right)^{L}
$$

which allows us to match the equation to (67) by using the relation between $u_{1, i}$ and the quasi-momentum $k_{i}$ ([6], eqn. (4.39)):

$$
k\left(u_{1, i}\right)=-i \log \frac{u_{1, i}+i / 2}{u_{1, i}-i / 2} .
$$

Note that the momenta and energy depend only on the $u_{1, i}$ excitations. For the $u_{q, i}, q \neq 1$ excitations, the left hand side of (68) becomes trivial, but the right hand side constrains the possible $u_{q, i}$. One should think of the $u_{1, i}$ as equivalent to the quasi-momenta $k_{i}$, and creating an actual excitation with energy and momentum, while the other $u_{q, i}$ do not change the energy or momentum but instead change the Lie group quantum numbers of the excitation.

The open chain Bethe ansatz equations generalize (67) in an intuitive way. In the open case, instead of making one complete circuit of the closed chain, picking up phases $S_{j i}$ for 
each interaction, a given excitation passes one way across the chain, reflects off one boundary, passes back the other way across the chain, and reflects off the other boundary:

$$
e^{2 i k_{i} L}=\mathcal{B}_{1}\left(k_{i}\right) \mathcal{B}_{2}\left(k_{i}\right) \prod_{j \neq i}\left(S_{j i}\left(k_{j}, k_{i}\right) S_{j i}\left(k_{j},-k_{i}\right)\right)
$$

Here $\mathcal{B}_{1}$ and $\mathcal{B}_{2}$ are phases generated by reflecting off the boundary, while the $S_{j i}$ are the same $S$-matrix terms as in the closed chain; the interaction between the spin waves is ignorant of the boundary conditions. We get each $S_{j i}$ twice because we pass both ways along the chain, with the relative momentum reversed between the two interactions.

So we see that if one already knows about the closed chain, the $S_{j i}$ are known, and determining the open chain Bethe ansatz equations comes down to finding $\mathcal{B}_{1}$ and $\mathcal{B}_{2}$. In our case the $S_{j i}$ are determined by (68) for group $S O(6)$, as was studied in [6]. The reflection coefficients $\mathcal{B}_{1}$ and $\mathcal{B}_{2}$ involve only the excitation in question and the boundary; whether there are other excitations is irrelevant. They can hence be determined by considering the case with just a single excitation. We do this below.

\subsection{Bethe Reference State}

We use as our Bethe reference state a particular chiral primary of the dCFT. Defining $Z \equiv X_{H}^{1}+i X_{H}^{2}$, we take

$$
|0\rangle_{L} \equiv \bar{q}_{1} Z Z \ldots Z q_{2}
$$

which results from (33) where for each $a=1 \ldots L+1$ one has chosen $I_{a}=+$, in the standard basis where $J_{3}$ is the Cartan generator of $S O(3)_{H}$. This state has $j_{H}=L+1, j_{V}=0$.

\subsection{Single Impurity Sector- $-S O(3)_{V}$ Fluctuation}

We now consider the case where any single $Z$ field in the chain is replaced by another $X^{i}$ such that $j_{H}=L .{ }^{4}$ There are two ways we can do this: we can excite one of the $S O(3)_{V}$ scalars, or we can excite $X_{H}^{3}$.

First consider an $S O(3)_{V}$ excitation; since the Bethe state is invariant under $S O(3)_{V}$, any of the three scalars are equivalent, and we will let $W$ be any of $X_{V}^{4}, X_{V}^{5}$, or $X_{V}^{6}$. We define the state $|W(x)\rangle$ of the spin chain as follows:

$$
|W(x)\rangle \equiv \bar{q}_{1} Z Z \ldots W \ldots Z q_{2}
$$

\footnotetext{
${ }^{4}$ Note that replacing a $Z$ by $\bar{Z}$ actually leads to $j_{H}=L-1$; this mixes with two $X^{3}$ impurities and hence will not be included in this section.
} 
where $W$ has replaced $Z$ at the $x^{t h}$ site. We then make a standard Bethe ansatz for the energy eigenstates $|W(k)\rangle$ :

$$
|W(k)\rangle \equiv \sum_{x=1}^{L} f(x)|W(x)\rangle, \quad f(x)=A(k) e^{i k x}+\tilde{A}(k) e^{-i k x}
$$

Unlike the closed chain ansatz, where we need consider waves propagating only in one direction, this case requires, because of reflection, that both directions be present. Integrability underlies the assumption that the magnitude $k$ of the momentum will not change, either in interacting with another excitation or in reflecting off the boundary.

Now we wish to determine the action of the Hamiltonian on the position eigenstates $|W(x)\rangle$; for convenience we work with $H$ defined as $\gamma_{\mathcal{O}} \equiv\left(g^{2} N / 16 \pi^{2}\right) H$. Consider first the case where $x \neq 1, L$; the boundary parts $2 I_{\bar{\alpha} 1}+R_{\bar{\alpha} 1}, 2 I_{L \beta}+2 S_{L \beta}$ of $H$ act on the same fields $\bar{q}_{1} Z$ and $Z q_{2}$ as in the chiral primary, and hence give zero. The same is true for $h_{j, j+1}$ acting on a pair $Z Z$. Hence the only nonzero contributions to the Hamiltonian are

$$
\begin{aligned}
h_{x, x+1}|W(x)\rangle & =\left(K_{x, x+1}+2 I_{x, x+1}-2 P_{x, x+1}|W(x)\rangle=0+2|W(x)\rangle-2|W(x+1)\rangle,\right. \\
h_{x-1, x}|W(x)\rangle & =\left(K_{x-1, x}+2 I_{x-1, x}-2 P_{x-1, x}|W(x)\rangle=0+2|W(x)\rangle-2|W(x-1)\rangle\right.
\end{aligned}
$$

leading to

$$
H|W(x)\rangle=4|W(x)\rangle-2|W(x-1)\rangle-2|W(x+1)\rangle .
$$

Now consider the states $|W(1)\rangle$ and $|W(L)\rangle$. Nonzero contributions are

$$
\begin{aligned}
h_{1,2}|W(1)\rangle=2|W(1)\rangle-2|W(2)\rangle, & \left(2 I_{\bar{\alpha} 1}+2 R_{\bar{\alpha} 1}\right)|W(1)\rangle=4|W(1)\rangle, \\
h_{L-1, L}|W(1)\rangle=2|W(L)\rangle-2|W(L-1)\rangle, & \left(2 I_{L \beta}+2 R_{L \beta}\right)|W(L)\rangle=4|W(L)\rangle .
\end{aligned}
$$

Thus we have

$$
H|W(1)\rangle=6|W(1)\rangle-2|W(2)\rangle, \quad H|W(L)\rangle=6|W(L)\rangle-2|W(L-1)\rangle .
$$

Now we demand that the states $|W(k)\rangle$ be energy eigenstates:

$$
(H-E(k))|W(k)\rangle=0,
$$

implying the relations

$$
\begin{aligned}
& 0=(4-E(k)) f(x)-2 f(x+1)-2 f(x-1), \quad 2 \leq x \leq L-1 \\
& 0=(6-E(k)) f(1)-2 f(2), \quad 0=(6-E(k)) f(L)-2 f(L-1) .
\end{aligned}
$$

Using the first relation we can deduce

$$
E(k)=4(1-\cos k) .
$$


We may then solve for $A(k)$ and $\tilde{A}(k)$ as follows. All the relations (81) are consistent with the single result (82) only if the exceptional second and third equations in (81) take the same form as the first, with auxiliary quantities $f(0), f(L+1)$ introduced. This follows only if

$$
f(0)=-f(1), \quad f(L+1)=-f(L) .
$$

If we plug this into the Bethe ansatz (74), these equations imply

$$
\begin{aligned}
A(k)\left(1+e^{i k}\right) & =-\tilde{A}(k)\left(1-e^{-i k}\right), \\
A(k) e^{i k L}\left(1+e^{i k}\right) & =-\tilde{A}(k) e^{-i k L}\left(1+e^{-i k}\right),
\end{aligned}
$$

which can be seen to have a solution only if

$$
e^{2 i k L}=1
$$

This is the Bethe ansatz equation for this impurity. We see that in fact the boundary interactions are trivial and an elementary quantization of the quasi-momentum $k$ is implied:

$$
\mathcal{B}_{1}=\mathcal{B}_{2}=1, \quad k=\frac{\pi n}{L}
$$

Meanwhile one finds the solution $A(k) / \tilde{A}(k)=-\left(1+e^{-i k}\right) /\left(1+e^{i k}\right)$. The energy eigenstates (74) can hence be written in terms of the integer $n$ :

$$
|W(n)\rangle=a_{n} \sum_{x=1}^{L} \sin \left[\frac{\pi n}{L}(x-1 / 2)\right]|W(x)\rangle
$$

up to some normalization $a_{n}$, with the associated anomalous dimension

$$
\gamma_{W(n)}=\frac{g^{2} N}{4 \pi^{2}}\left[1-\cos \left(\frac{\pi n}{L}\right)\right] .
$$

We see that the boundary conditions imposed on the spin waves are that they go to zero at the "end points" a half-step past the last links on the chain; these are Dirichlet boundary conditions.

This is precisely what we expect from the dual string theory point of view. In the string dual, this operator corresponds to an open string living on a D5-brane; and oscillations in the three $S O(3)_{V}$ directions normal to the brane must obey Dirichlet boundary conditions at the endpoints. Moreover, the periodicity (86) with no $\mathcal{B}$ factors tells us that the left and right movers on the chain can be recast as just left moving excitations on a closed chain of length $2 L$; this is nothing but the familiar "doubling trick" of open string theory. We now turn to a discussion of the $X^{3}$ excitation, which should be associated with Neumann boundary conditions. 


\subsection{Single Impurity Sector $-X^{3}$ Fluctuation}

We now consider an $X^{3}$ impurity, which we will abbreviate as just $X$. This lowers the $j_{H}$ charge to $j_{H}=L$ without turning on a $j_{V}$ charge. As before we define states with a single impurity

$$
|X(x)\rangle \equiv \bar{q}_{1} Z Z \ldots X \ldots Z q_{2}
$$

with $X$ replacing $Z$ at site $x$. However, these states are not a closed set under action of the Hamiltonian. We may also create states with $j_{H}=L, j_{V}=0$ by flipping the spin of one of the $q$ fields on the end of the chain, but leaving all bulk excitations as $Z$ :

$$
\left|q_{1}\right\rangle \equiv \bar{q}_{1} Z Z \ldots Z q_{1}, \quad\left|\bar{q}_{2}\right\rangle \equiv \bar{q}_{2} Z Z \ldots Z q_{2}
$$

We assume the energy eigenstates to be of the form

$$
|X(k)\rangle \equiv \sum_{x=1}^{L} g(x)|X(x)\rangle+\alpha\left|q_{1}\right\rangle+\beta\left|\bar{q}_{2}\right\rangle, \quad g(k)=B(k) e^{i k x}+\tilde{B}(k) e^{-i k x}
$$

The analysis then follows as before. Requiring $|X(k)\rangle$ to be an energy eigenstate

$$
(H-E(k))|X(k)\rangle=0
$$

leads to the equations

$$
\begin{aligned}
& 0=(4-E) \beta+2 g(1), \\
& 0=(4-E) g(1)+4 \beta-2 g(2), \\
& 0=(4-E) g(x)-2 g(x-1)-2 g(x+1), \quad 2 \leq x \leq L-1, \\
& 0=(4-E) g(L)-2 g(L-1)-4 \alpha, \\
& 0=(4-E) \alpha-2 g(L) .
\end{aligned}
$$

The energy has the same form as in the previous subsection, as can be deduced from the generic $(2 \leq x \leq L-1)$ equation

$$
E(k)=4(1-\cos k) .
$$

Again we proceed by attempting to cast the other equations in this same form. For the second and fourth equations, this can be done using the definitions

$$
g(0) \equiv-2 \beta, \quad g(L+1) \equiv 2 \alpha .
$$

The first and last equations also share this form if we introduce the fictitious

$$
g(-1) \equiv g(1), \quad g(L+2) \equiv g(L)
$$


These relations imply for the ansatz (92)

$$
\begin{aligned}
B(k)\left(e^{-i k}-e^{i k}\right) & =\tilde{B}(k)\left(e^{-i k}-e^{i k}\right) \\
B(k) e^{i k L}\left(e^{2 i k}-1\right) & =\tilde{B}(k) e^{-i k L}\left(1-e^{-2 i k}\right) .
\end{aligned}
$$

The first obviously forces $B(k)=\tilde{B}(k)$. The second is then consistent only if

$$
e^{2 i k(L+1)}=1, \quad k=\frac{\pi n}{L+1} .
$$

Once again the Bethe ansatz equation indicates that the boundary reflection coefficients are trivial: $\mathcal{B}_{1}=\mathcal{B}_{2}=1$. The chain is effectively one link longer here, because of the participation of the $q$ fields in the spin wave. The energy eigenstates can be written

$$
|X(n)\rangle=b_{n} \sum_{x=0}^{L+1} \cos \left(\frac{\pi n x}{L+1}\right)|X(x)\rangle,
$$

where we have defined

$$
|X(0)\rangle \equiv-(1 / 2)\left|\bar{q}_{2}\right\rangle, \quad|X(L+1)\rangle \equiv(1 / 2)\left|q_{1}\right\rangle
$$

and the anomalous dimension is

$$
\gamma_{W(n)}=\frac{g^{2} N}{4 \pi^{2}}\left[1-\cos \left(\frac{\pi n}{L+1}\right)\right]
$$

We notice immediately that for these excitations, the boundary conditions are Neumann. Since $X$ corresponds to a fluctuation in a direction in which the brane is extended, this is again exactly what we expect from the dual string point of view; the possibility of flipping the spin on the $q$ fields plays a fundamental role in allowing the motion of the endpoints. Again the left and right movers together behave as a single closed chain, this time of length $2(L+1)$.

We conclude that the integrability of the system allows us to see with particular clarity the emergence of open strings from the defect local operators. Oscillations of the string are nothing but spin waves propagating on the chain, reflecting with boundary conditions that are equivalent to trivial transmission onto a "doubled" closed chain.

\subsection{Plane Wave Limit}

The plane wave limit of the gravity dual to the dCFT was studied by Lee and Park [33]. They considered a Penrose limit where the defining null geodesic wound around the $S^{2} \subset S^{5}$ on which the D5-brane is wrapped; the associated light-cone vacuum $\left|0, p^{+}\right\rangle$is just our Bethe reference state, the chiral primary $|0\rangle_{L}$, with $\left(\mu p^{+} \alpha^{\prime}\right)^{2}=J^{2} /\left(g^{2} N\right), J=L$. They 
furthermore studied single-impurity excitations, and it is interesting to match these to our results.

Lee and Park identified an open string excitation in a Dirichlet direction having oscillator number $n$ with the operator

$$
a_{n}^{\dagger, D}\left|0, p^{+}\right\rangle \quad \leftrightarrow \quad \frac{1}{\sqrt{J}} \sum_{0}^{J} \frac{\sqrt{2} \sin \left(\frac{\pi n l}{J}\right)}{N^{J / 2+1}} \bar{q}_{1} Z^{l} X^{3} Z^{J-l} q_{2},
$$

while a Neumann direction excitation took the form ${ }^{5}$

$$
a_{n}^{\dagger, N}\left|0, p^{+}\right\rangle \quad \leftrightarrow \quad \frac{1}{\sqrt{J}} \sum_{0}^{J} \frac{\sqrt{2} \cos \left(\frac{\pi n l}{J}\right)}{N^{J / 2+1}} \bar{q}_{1} Z^{l} X^{3} Z^{J-l} q_{2} .
$$

They identified the anomalous dimension for both cases as

$$
\gamma_{\text {plane }}=\frac{\pi g_{s} N n^{2}}{2 J^{2}}
$$

Using the relation $4 \pi g_{s}=g^{2}$, we find that the large- $L$ limit of our results indeed agrees with the conclusions (108)-(110) of [33] for the plane wave limit.

\section{Conclusions and Open Questions}

The presence of integrability inside four dimensional quantum field theories is unexpected and fascinating. We have seen that the sector of open chain operators constructed from scalar fields in the superconformal defect deformation of $\mathcal{N}=4$ Super Yang-Mills possesses an integrable structure, taking the form of integrable boundary conditions added to the structure of $\mathcal{N}=4 \mathrm{SYM}$ alone. It is natural to hypothesize that this integrability holds for all operators in the dCFT. Given that theories closely related to $\mathcal{N}=4$ are also integrable, one may speculate on how broad a class of theories possesses an integrable structure, and to what extent integrability is a useful concept for understanding other, non-conformal gauge theories, for example those that confine.

The existence of the open chains also implies that in the gravity dual, open string boundary conditions on D-branes can be integrable. It is natural to suspect that an $O S p(4 \mid 4)$ Yangian will survive in the open Green-Schwarz worldsheet theory. It would be interesting to verify the presence of this symmetry, and to look for other D-brane configurations that preserve integrability.

Finally, we have seen in the interactions of the spin waves in the Bethe ansatz how properties of open strings like Dirichlet or Neumann boundary conditions and the doubling trick

\footnotetext{
${ }^{5}$ The authors of [33] mention that operators analogous to (91) with $q$ spins flipped could mix with (109), but do not include them explicitly.
} 
arise in the field theory dual as an open string is assembled from "bits" of quantum fields on the spin chain. In this sense integrability, like supersymmetry, provides a crutch to help us see the workings of duality. It would be interesting to use the Bethe ansatz to diagonalize more complicated states so as to learn more about mesonic-type operators and their properties under duality, and in greater generality to learn more about how strings are assembled from field theory constituents.

\section{Acknowledgments}

We benefited from discussions with David Berenstein, Neil Constable, Chris Herzog, John McGreevy, Joe Polchinski, Radu Roiban, Marcus Spradlin, Herman Verlinde, Anastasia Volovich, and Johannes Walcher. This material is based upon work supported by the National Science Foundation under grants Nos. 0243680 (O.D.) and PHY00-98395 (N.M.). Any opinions, findings, and conclusions or recommendations expressed in this material are those of the authors and do not necessarily reflect the views of the National Science Foundation. The work of N.M. was also supported by a National Defense Science and Engineering Graduate Fellowship.

\section{Appendix: Field Theory Conventions}

Our index conventions are as follows. $\mu, \nu$ and $k, l$ are $4 \mathrm{D}$ and 3D Lorentz indices. We denote the $\mathbf{6}$ of $S O(6)_{R}$ with $i, j$; it decomposes under $S O(3)_{H} \times S O(3)_{V}$ as $\mathbf{6} \rightarrow(\mathbf{3}, \mathbf{1}) \oplus(\mathbf{1}, \mathbf{3})$, labeled with $I, J$ and $A, B$, respectively. The 4 of $S O(6)_{R}$ is $\alpha, \beta$, and it decomposes as $\mathbf{4} \rightarrow(\mathbf{2}, \mathbf{2})$; doublets of $S O(3)_{H}$ are denoted $m, n$ and doublets of $S O(3)_{V}$ are $a, b$.

We work in Euclidean space rotated from mostly-minus signature, where the $\gamma$-matrix conventions are those of [31, and are such that Majorana spinors are real.

We write our adjoint fields in matrix notation, and color indices are implicit throughout. The action for $\mathcal{N}=4 \mathrm{SYM}$ is

$$
S_{4}=\frac{1}{g^{2}} \int d^{4} x \operatorname{Tr}\left(\frac{1}{2} F_{\mu \nu} F^{\mu \nu}-i \bar{\lambda}^{\alpha} \gamma^{\mu} D_{\mu} \lambda^{\alpha}+D_{\mu} X^{a} D^{\mu} X^{a}-\frac{1}{2}\left[X^{a}, X^{b}\right]+C_{\alpha \beta}^{a} \bar{\lambda}^{\alpha}\left[X^{a}, \lambda^{\beta}\right]\right)(11
$$

with the covariant derivative $D_{\mu} X^{a}=\partial_{\mu} X^{a}-i\left[A_{\mu}, X^{a}\right]$ and similarly for $\lambda^{\alpha}$.

The Yukawa tensor $C_{\alpha \beta}^{a}$ in the $\mathbf{4} \times \mathbf{4} \rightarrow \mathbf{6}$ can be written explicitly as follows. Split the fermions as $\chi^{A} \equiv \lambda^{\alpha}, A, \alpha=1,2,3$, and $\lambda \equiv \lambda^{4}$, and assemble complex scalars as $\Phi^{A} \equiv\left(X_{V}^{A}+i X_{H}^{A}\right) \sqrt{2}$ with $X_{V}^{1,2,3} \equiv X^{1,2,3}$ and $X_{H}^{1,2,3} \equiv X^{4,5,6}$. Then

$$
\begin{aligned}
& \int d^{4} x \operatorname{Tr} C_{\alpha \beta}^{a} \bar{\lambda}^{\alpha}\left[X^{a}, \lambda^{\beta}\right]= \\
& i 2 \sqrt{2} \int d^{4} x \operatorname{Tr}\left(\bar{\lambda}\left[L \chi^{A}, \bar{\Phi}^{A}\right]-\bar{\chi}^{A}\left[R \lambda, \Phi^{A}\right]-\frac{1}{2} \epsilon_{A B C} \bar{\chi}^{A}\left[\left(L \Phi^{C}+R \bar{\Phi}^{C}\right), \chi^{B}\right]\right),
\end{aligned}
$$


with $L \equiv\left(1+\gamma^{5}\right) / 2, R \equiv\left(1-\gamma^{5}\right) / 2$.

The $\lambda^{\alpha}$ are arranged into $\lambda_{a m}$ transforming in the $(\mathbf{2}, \mathbf{2})$ of $S O(3)_{H} \times S O(3)_{V}$ :

$$
\lambda_{i m} \equiv \lambda \delta_{i m}-i \chi^{A} \sigma_{i m}^{A} .
$$

We use $z_{\mu}$ to indicate $4 \mathrm{D}$ coordinates and decompose them into 3D coordinates $y_{k}$ and transverse $x \equiv x_{3}$. The massless $4 \mathrm{D}$ and 3D scalar propagators in Feynman gauge are

$$
\begin{aligned}
\left\langle X\left(z_{1}\right) X\left(z_{2}\right)\right\rangle=\frac{1}{2} \Delta_{12} \equiv \frac{1}{2} \frac{1}{4 \pi^{2}} \frac{1}{\left(z_{12}\right)^{2}}, \quad\left\langle A_{\mu}\left(z_{1}\right) A_{\nu}\left(z_{2}\right)\right\rangle=\frac{1}{2} \delta_{\mu \nu} \Delta_{12}, \\
\left\langle\lambda\left(z_{1}\right) \bar{\lambda}\left(z_{2}\right)\right\rangle \equiv \frac{1}{2} S_{12}=\frac{1}{2} \gamma^{\mu} \partial_{\mu} \Delta_{12}, \quad\left\langle q\left(y_{1}\right) \bar{q}\left(y_{2}\right)\right\rangle=\mathcal{D}_{12} \equiv \frac{1}{4 \pi} \frac{1}{\left|y_{12}\right|},
\end{aligned}
$$

where we have suppressed the color delta functions; the factors of $1 / 2$ arise for the $4 \mathrm{D}$ fields because of the matrix notation we are using. Since $\lambda$ is Majorana, we also have non-vanishing $\left\langle\lambda\left(z_{1}\right) \lambda\left(z_{2}\right)\right\rangle$ and $\left\langle\bar{\lambda}\left(z_{1}\right) \bar{\lambda}\left(z_{2}\right)\right\rangle$, determined by $\bar{\lambda}=\lambda^{T} \gamma^{0}$.

We write the $3 \mathrm{D}$ fermion $\Psi$ in a $4 \mathrm{D}$ notation, as a four-component spinor satisfying

$$
P_{+} \Psi=\Psi, \quad P_{-} \Psi=0, \quad P_{ \pm} \equiv \frac{1}{2}\left(1 \pm \gamma^{5} \gamma^{3}\right) ，
$$

where $\gamma^{5} \equiv-i \gamma^{0} \gamma^{1} \gamma^{2} \gamma^{3}$ is the chirality matrix. The propagator for $\Psi$ is then

$$
\left\langle\Psi\left(z_{1}\right) \bar{\Psi}\left(z_{2}\right)\right\rangle=\left(\gamma^{k} P_{+}\right) \partial_{k} \mathcal{D}_{12} \equiv \hat{s}_{12} .
$$

The projection matrices $P_{ \pm}$obey

$$
\left[P_{ \pm}, \gamma^{k}\right]=0, \quad \gamma^{3} P_{ \pm}=P_{\mp} \gamma^{x}, \quad P_{+}^{2}=P_{-}^{2}=1, \quad P_{+} P_{-}=0 .
$$

Propagators for $\lambda_{i m}$ can be written in terms of (114) as

$$
\begin{aligned}
\left\langle\lambda_{i m}\left(z_{1}\right) \bar{\lambda}_{n j}\left(z_{2}\right)\right\rangle & =2\left\langle\lambda\left(z_{1}\right) \bar{\lambda}\left(z_{2}\right)\right\rangle \delta_{i j} \delta_{m n}, \\
\left\langle\lambda_{i m}\left(z_{1}\right) \lambda_{j n}\left(z_{2}\right)\right\rangle & =2\left\langle\lambda\left(z_{1}\right) \lambda\left(z_{2}\right)\right\rangle \epsilon_{i j} \epsilon_{m n}, \\
\left\langle\bar{\lambda}_{m i}\left(z_{1}\right) \bar{\lambda}_{n j}\left(z_{2}\right)\right\rangle & =2\left\langle\bar{\lambda}\left(z_{1}\right) \bar{\lambda}\left(z_{2}\right)\right\rangle \epsilon_{i j} \epsilon_{m n} .
\end{aligned}
$$

In differential regularization we use the replacements (neglecting quadratic divergences):

$$
\begin{aligned}
& \frac{1}{z^{4}} \rightarrow-\frac{1}{4} \text { ㅁ } \frac{\log z^{2} M^{2}}{z^{2}}, \\
& \frac{1}{z^{6}} \rightarrow-\frac{1}{32} \text { ㅁ } \frac{\log z^{2} M^{2}}{z^{2}} \text {, } \\
& \frac{1}{|y|^{3}} \rightarrow-\nabla^{2} \frac{\log M|y|}{|y|}, \\
& \frac{1}{|y|^{5}} \rightarrow-\frac{1}{6} \nabla^{2} \nabla^{2} \frac{\log M|y|}{|y|},
\end{aligned}
$$

where $\square$ and $\nabla^{2}$ are the 4D and 3D Laplacians, respectively. 


\section{References}

[1] J. M. Maldacena, "The Large N Limit of Superconformal Field Theories and Supergravity," Adv. Theor. Math. Phys. 2, 231 (1998) [Int. J. Theor. Phys. 38, 1113 (1999)] arXiv:hep-th/9711200.

[2] S. S. Gubser, I. R. Klebanov and A. M. Polyakov, "Gauge Theory Correlators from Non-Critical String Theory," Phys. Lett. B 428, 105 (1998) arXiv:hep-th/9802109.

[3] E. Witten, "Anti-de Sitter Space and Holography," Adv. Theor. Math. Phys. 2, 253 (1998) arXiv:hep-th/9802150.

[4] O. Aharony, S. S. Gubser, J. M. Maldacena, H. Ooguri and Y. Oz, "Large N field Theories, String Theory and Gravity," Phys. Rept. 323, 183 (2000) arXiv:hep-th/9905111.

[5] D. Berenstein, J. M. Maldacena and H. Nastase, "Strings in Flat Space and Pp Waves from N = 4 Super Yang Mills," JHEP 0204, 013 (2002) arXiv:hep-th/0202021.

[6] J. A. Minahan and K. Zarembo, "The Bethe-Ansatz for $\mathcal{N}=4$ Super Yang Mills," JHEP 0303 (2003) 013 arXiv:hep-th/0212208.

[7] N. Beisert and M. Staudacher, "The N = 4 SYM Integrable Super Spin Chain," Nucl.Phys. B670, (2003) 439-463 arXiv:hep-th/0307042.

[8] I. Bena, J. Polchinski and R. Roiban, "Hidden Symmetries of the $A d S_{5} \times S^{5}$ Superstring," arXiv:hep-th/0305116.

[9] L. Dolan, C. R. Nappi, E. Witten, "A Relation Between Approaches to Integrability in Superconformal Yang-Mills Theory" JHEP 0310 (2003) 017 arXiv:hep-th/0308089.

[10] A. V. Belitsky, A. S. Gorsky and G. P. Korchemsky, "Gauge / string duality for QCD conformal operators," Nucl. Phys. B 667, 3 (2003) arXiv:hep-th/0304028.

[11] N. Beisert, J. A. Minahan, M. Staudacher and K. Zarembo, "Stringing Spins and Spinning Strings," JHEP 0309, 010 (2003) arXiv:hep-th/0306139.

[12] B. C. Vallilo, "Flat Currents in the Classical AdS(5) x S**5 Pure Spinor Superstring," arXiv:hep-th/0307018.

[13] G. Arutyunov, S. Frolov, J. Russo and A. A. Tseytlin, "Spinning Strings in AdS(5) x S**5 and Integrable Systems," Nucl. Phys. B 671, 3 (2003) arXiv:hep-th/0307191.

[14] N. Beisert, "Higher Loops, Integrability and the Near BMN Limit," JHEP 0309, 062 (2003) arXiv:hep-th/0308074. 
[15] N. Beisert, S. Frolov, M. Staudacher and A. A. Tseytlin, "Precision Spectroscopy of AdS/CFT," JHEP 0310, 037 (2003) arXiv:hep-th/0308117.

[16] A. Gorsky, "Spin Chains and Gauge/String Duality," arXiv:hep-th/0308182.

[17] L. F. Alday, "Nonlocal Charges on $\operatorname{AdS}(5)$ x S**5 and Pp-Waves," JHEP 0312, 033 (2003) arXiv:hep-th/0310146.

[18] G. Arutyunov and M. Staudacher, "Matching Higher Conserved Charges for Strings and Spins," arXiv:hep-th/0310182.

[19] J. Engquist, J. A. Minahan and K. Zarembo, "Yang-Mills Duals for Semiclassical Strings on $\operatorname{AdS}(5)$ x S**5," JHEP 0311, 063 (2003) arXiv:hep-th/0310188.

[20] T. Klose and J. Plefka, "On the Integrability of Large N Plane-Wave Matrix Theory," arXiv:hep-th/0310232.

[21] N. Beisert, "The su(2|3) Dynamic Spin Chain," arXiv:hep-th/0310252.

[22] G. Arutyunov, J. Russo and A. A. Tseytlin, "Spinning Strings in AdS(5) x S**5: New Integrable System Relations," arXiv:hep-th/0311004.

[23] A. Mikhailov, "Speeding Strings," arXiv:hep-th/0311019.

[24] A. V. Belitsky, S. E. Derkachov, G. P. Korchemsky and A. N. Manashov, "Superconformal operators in $\mathrm{N}=4$ super-Yang-Mills theory," arXiv:hep-th/0311104.

[25] A. A. Tseytlin, "Spinning Strings and AdS/CFT Duality," arXiv:hep-th/0311139.

[26] M. Kruczenski, "Spin Chains and String Theory," arXiv:hep-th/0311203.

[27] B. J. Stefanski, "Open spinning strings," arXiv:hep-th/0312091.

[28] N. W. Kim, "Multi-spin strings on $\operatorname{AdS}(5) \times \mathrm{T}^{* *}(1,1)$ and operators of $\mathrm{N}=1$ superconformal theory," arXiv:hep-th/0312113.

[29] X. J. Wang and Y. S. Wu, "Integrable Spin Chain and Operator Mixing in N = 1,2 Supersymmetric Theories," arXiv:hep-th/0311073.

[30] R. Roiban, "On Spin Chains and Field Theories," arXiv:hep-th/0312218.

[31] O. DeWolfe, D. Z. Freedman, and H. Ooguri, "Holography and Defect Conformal Field Theory," Phys.Rev. D66 (2002) 025009 arXiv:hep-th/0111135.

[32] J. Erdmenger, Z. Guralnik and I. Kirsch, "Four-Dimensional Superconformal Theories with Interacting Boundaries or Defects," Phys. Rev. D 66, 025020 (2002) arXiv:hep-th/0203020. 
[33] P. Lee and J. Park, "Open Strings in PP-Wave Background from Defect Conformal Field Theory," Phys.Rev. D67 (2003) 026002 arXiv:hep-th/0203257.

[34] K. Skenderis and M. Taylor, "Branes in AdS and Pp-Wave Spacetimes," JHEP 0206, 025 (2002) arXiv:hep-th/0204054.

[35] A. Karch and E. Katz, "Adding Flavor to AdS/CFT," JHEP 0206, 043 (2002) arXiv:hep-th/0205236.

[36] D. Mateos, S. Ng and P. K. Townsend, "Supersymmetric Defect Expansion in CFT from AdS Supertubes," JHEP 0207, 048 (2002) arXiv:hep-th/0207136.

[37] S. Yamaguchi, "Holographic RG Flow on the Defect and g-Theorem," JHEP 0210, 002 (2002) arXiv:hep-th/0207171.

[38] N. R. Constable, J. Erdmenger, Z. Guralnik and I. Kirsch, "Intersecting D3-branes and Holography," Phys. Rev. D 68, 106007 (2003) arXiv:hep-th/0211222.

[39] N. R. Constable, J. Erdmenger, Z. Guralnik and I. Kirsch, "(De)Constructing Intersecting M5-Branes," Phys. Rev. D 67, 106005 (2003) arXiv:hep-th/0212136.

[40] N. R. Constable, J. Erdmenger, Z. Guralnik and I. Kirsch, "Intersecting Branes, Defect Conformal Field Theories and Tensionless Strings," Fortsch. Phys. 51, 732 (2003) arXiv:hep-th/0212265.

[41] O. Aharony, O. DeWolfe, D. Z. Freedman and A. Karch, "Defect Conformal Field Theory and Locally Localized Gravity," JHEP 0307, 030 (2003) arXiv:hep-th/0303249.

[42] D. Bak, M. Gutperle and S. Hirano, "A Dilatonic Deformation of AdS(5) and its Field Theory Dual," JHEP 0305, 072 (2003) arXiv:hep-th/0304129.

[43] S. Yamaguchi, "AdS Branes Corresponding to Superconformal Defects," JHEP 0306, 002 (2003) arXiv:hep-th/0305007.

[44] J. Babington, J. Erdmenger, N. Evans, Z. Guralnik and I. Kirsch, "Chiral Symmetry Breaking and Pions in Non-Supersymmetric Gauge/Gravity Duals," arXiv:hep-th/0306018.

[45] J. Erdmenger, Z. Guralnik, R. Helling and I. Kirsch, "A World-Volume Perspective on the Recombination of Intersecting Branes," arXiv:hep-th/0309043.

[46] M. Kruczenski, D. Mateos, R. C. Myers and D. J. Winters, "Towards a Holographic Dual of Large-N(c) QCD," arXiv:hep-th/0311270.

[47] J. Babington, J. Erdmenger, N. Evans, Z. Guralnik and I. Kirsch, "A Gravity Dual of Chiral Symmetry Breaking," arXiv:hep-th/0312263. 
[48] A. Karch and L. Randall, "Localized Gravity in String Theory," Phys. Rev. Lett. 87, 061601 (2001) arXiv:hep-th/0105108.

[49] A. Karch and L. Randall, "Open and Closed String Interpretation of SUSY CFT's on Branes with Boundaries," JHEP 0106, 063 (2001) arXiv:hep-th/0105132.

[50] A. Fayyazuddin, "Supersymmetric Webs of D3/D5-Branes in Supergravity," JHEP 0303, 033 (2003) arXiv:hep-th/0207129.

[51] S. A. Cherkis and A. Hashimoto, "Supergravity Solution of Intersecting Branes and AdS/CFT with Flavor," JHEP 0211, 036 (2002) arXiv:hep-th/0210105.

[52] D. Arnaudon, J. Avan, N. Crampe, A. Doikou, L. Frappat and E. Ragoucy, "Classification of reflection matrices related to (super) Yangians and application to open spin chain models," Nucl. Phys. B 668, 469 (2003) arXiv:math.qa/0304150.

[53] D. Arnaudon, J. Avan, N. Crampe, A. Doikou, L. Frappat and E. Ragoucy, "Bethe Ansatz equations and exact $\mathrm{S}$ matrices for the $\operatorname{osp}(\mathrm{M} \mid 2 \mathrm{n})$ open super spin chain," arXiv:math-ph/0310042.

[54] B. Chen, X. J. Wang and Y. S. Wu, "Integrable Open Spin Chain in Super Yang-Mills and the Plane-wave/SYM duality," arXiv:hep-th/0401016.

[55] V. M. Braun, S. E. Derkachov and A. N. Manashov, "Integrability of three-particle evolution equations in QCD," Phys. Rev. Lett. 81, 2020 (1998) arXiv:hep-ph/9805225.

[56] V. M. Braun, S. E. Derkachov, G. P. Korchemsky and A. N. Manashov, "Baryon distribution amplitudes in QCD," Nucl. Phys. B 553, 355 (1999) arXiv:hep-ph/9902375.

[57] A. V. Belitsky, "Fine structure of spectrum of twist-three operators in QCD," Phys. Lett. B 453, 59 (1999) arXiv:hep-ph/9902361.

[58] A. V. Belitsky, "Integrability and WKB solution of twist-three evolution equations," Nucl. Phys. B 558, 259 (1999) arXiv:hep-ph/9903512.

[59] A. V. Belitsky, "Renormalization of twist-three operators and integrable lattice models," Nucl. Phys. B 574, 407 (2000) arXiv:hep-ph/9907420.

[60] S. E. Derkachov, G. P. Korchemsky and A. N. Manashov, "Evolution equations for quark gluon distributions in multi-color QCD and open spin chains," Nucl. Phys. B 566, 203 (2000) arXiv:hep-ph/9909539.

[61] D. J. Gross, A. Mikhailov and R. Roiban, "Operators with Large R Charge in N $=4$ Yang-Mills Theory," Annals Phys. 301, 31 (2002) arXiv:hep-th/0205066. 
[62] C. Kristjansen, J. Plefka, G. W. Semenoff and M. Staudacher, "A New Double-Scaling Limit of $N=4$ Super Yang-Mills Theory and PP-Wave Strings," Nucl. Phys. B 643, 3 (2002) arXiv:hep-th/0205033.

[63] N. R. Constable, D. Z. Freedman, M. Headrick, S. Minwalla, L. Motl, A. Postnikov and W. Skiba, "PP-Wave String Interactions from Perturbative Yang-Mills Theory," JHEP 0207, 017 (2002) arXiv:hep-th/0205089.

[64] D. Z. Freedman, K. Johnson and J. I. Latorre, "Differential Regularization and Renormalization: A New Method of Calculation in Quantum Field Theory," Nucl. Phys. B 371, 353 (1992).

[65] E. K. Sklyanin, "Boundary Conditions For Integrable Quantum Systems," J. Phys. A 21, 2375 (1988).

[66] A. Doikou and R. I. Nepomechie, "Bulk and Boundary S Matrices for the $S U(N)$ Chain," Nucl.Phys. B521 (1998) 547-572 arXiv:hep-th/9803118.

[67] C. Gomez, M. Ruiz-Altaba and G. Sierra, Quantum Groups in Two-Dimensional Physics, Cambridge University Press, 1996. 